\title{
Therapeutic Effect of The Combination of Short-Term Massage Manipulation With Remote Guided Self-Exercise On The Post-Mastectomy Axillary Web Syndrome In Patients With Breast Cancer: A Randomized, Single-Blind Trial.
}

Jia Qiao

The First Affiliated Hospital of Chongqing Medical University

Qi-hui Yang

The First Affiliated Hospital of Chongqing Medical University

Jing Zhan

The First Affiliated Hospital of Chongqing Medical University

YI Li

The First Affiliated Hospital of Chongqing Medical University

Ding-qun Bai ( $\triangle$ Baidingqun2014@163.com)

The First Affiliated Hospital of Chongqing Medical University

Yu-han Kong

The First Affiliated Hospital of Chongqing Medical University

\section{Research Article}

Keywords: axillary web syndrome, AWS, massage manipulation, breast cancer

Posted Date: August 13th, 2021

DOI: https://doi.org/10.21203/rs.3.rs-726740/v1

License: @ (1) This work is licensed under a Creative Commons Attribution 4.0 International License. Read Full License 


\section{Abstract}

Background: Although the axillary web syndrome (AWS) is a common post-mastectomy complication. A small number of studies have reported treatment strategies for the AWS that showed interventions for one month were an effective strategy. The current study in AWS patients investigated the clinical efficacy of short-term massage manipulation (STMM) combined with remote guided self-exercise (RGS).

Method: The patients were randomized into either short-term massage manipulation combine with remote guided self-exercise group (STMM+RGS group) or treatment without massage manipulation and remote guided self-exercise group (TSTMM+RGS group). STMM+RGS group received massage manipulation for 20 min per day for 7 days as total hospitalized therapy and then received telephone-guided therapy for 9 weeks, rehabilitation gymnastics provided during hospitalization.TSTMM+RGS group received rehabilitation gymnastics only. The primary outcome was changes in upper limb range of movement (ROM), assessed at 3, 6, and 9 weeks after intervention. The secondary outcomes were scores of visual analog scales (VAS), disability of arm, shoulder and hand (DASH) index, and activities of daily living (ADL) index assessed at $3,6,9$ weeks of follow-up.

Results: 106 patients were enrolled in the trial, 86 were randomized to treatment groups, with 79 completing the trial (41 in STMM+RGS group, 38 in T-STMM+RGS group). At 6 and 9 weeks of follow-up ROM had improved in STMM+RGS group compared with that observed in T-STMM+RGS group $(p<0.05)$. This improvement was more pronounced and significantly different at 9 weeks $(p<0.01)$.. There was also a significant difference in VAS at 6 and 9 weeks between the two groups. The DASH score improved significantly in STMM+RGS group compared with that in T-STMM+RGS group at 3, 6, and 9 weeks of follow-up $(p<0.01)$, while the ADL score was significantly different $(p<0.05)$ in the two groups at 3 and 6 weeks, but showed no significant difference after 9 weeks of follow-up.

Conclusion: The combination of STMM and RGS improves upper limb ROM, relieves upper limb pain, and reduces the occurrence of disabilities in post-mastectomy patients with the AWS.

\section{Introduction}

Breast cancer is currently one of the most common malignant tumors in women. According to statistics, there are 279,000 new cases of breast cancer in Chinese women every year, with this number increasing year by year[1]. Sentinel lymph node biopsies (SLNB) and axillary lymph node dissection (ALND) are the main surgical procedures used to treat breast cancer in recent years[2, 3], although both procedures are often accompanied by complications, the most common being breast cancerrelated lymphedema (BCRL) and the axillary web syndrome (AWS)[4].

The AWS presents as a common post-mastectomy complication with an incidence rate of up to $85.4 \%[5]$, with the incidence higher with $\operatorname{ALND}(5.2-85.4 \%)$ than with $\operatorname{SLND}(0.9-20 \%[6,7]$. However, the incidence rate reported in the literature may vary because of different criteria used for evaluation.

AWS is characterized by 'cording or subcutaneous nodules' in the axillary, upper limbs, and even the chest wall(Picture1). It is often accompanied by pain in the ipsilateral upper limb, limited range of motion(ROM) in the shoulder and sometimes numbness in the upper limb[8-11]. The onset of AWS occurs mainly between 1-5 weeks after surgery, with most cases resolving within 2-3 months[12]. Because of the insidious onset of AWS it is often ignored by patients and clinicians. Most patients do not receive active treatment despite studies showing that cording can last for more than one year[13] and may even cause severe shoulder dysfunction[14-17]. Breast cancer patients need to undergo radiotherapy after surgery. This procedure requires the affected limb to be lifted above the head, and because AWS affects shoulder joint mobility, this results in patients being unable to maintain the position, seriously affecting the treatment effect of radiotherapy. Early intervention in AWS is therefore indispensable[4, 18]. 
The methods for treating AWS are limited. The main treatment method is rehabilitation manipulative therapy, supplemented by drug therapy. Common manipulative therapy includes soft tissue techniques[19] and manual lymphatic drainage[20]. Previous studies have reported that rehabilitation interventions lasting for a month are effective for treating AWS[20]. However in our experience patients show poor medical compliance with this type of intervention. The current study investigated a compromised intervention, that is short-term massage manipulation (STMM) combined with remote guided self-exercise (RGS). Massage manipulation was based on the findings of previous research and involved an improved method to treat the symptoms of AWS in the affected limbs by promoting lymphatic drainage using massaging strips. The procedure was performed for 20mins each day for a total of 7 days as hospitalized therapy, a shorter period than that used in previous research, and then continued during the follow-up period that used remote telephone contact to guide rehabilitation. Several variables were measured before and after the treatment to assess the clinical efficacy of STMM + RGS, including ROM and the scores of visual analog scales (VAS), disability of arm, shoulder and hand (DASH), and activities of daily living (ADL) questionnaires.

\section{Method}

\section{Study design and setting}

This study was designed as a randomized, single-blind trial of two different intervention (with or without massage manipulation and remotely guided treatment) for post-mastectomy AWS. All the patients were from the Department of Endocrinology and Breast Surgery, the First Affiliated Hospital of Chongqing Medical University, and received oral and written information about the study and written informed consent forms while enroll in the study. The study has been approved by the Ethics Committee of the First Affiliated Hospital of Chongqing Medical University and registered in the clinical trial registration center, the registration number is ChiCTR1900028060.

\section{Patients}

Patients were enrolled in the study from October 2019 to October 2020, with their eligibility assessed by experienced physiotherapists. A total of 86 patients were randomly divided into either STMM + RGS group or T-STMM + RGS group using a random number table. In STMM + RGS group, the short-term massage manipulation combined with remote guided therapy was provided by telephone calls from physiotherapists, and rehabilitation gymnastics during hospitalization was provided by nurses. The patients in T-STMM + RGS group only received rehabilitation gymnastics during hospitalization.

The inclusion criteria for the study were: 1. Patients who had received ALND or SLND surgery; 2. Patients diagnosed with AWS confirmed by inspection and palpation; 3. Patients who agreed to complete the relevant evaluation scales and investigations; 4. No other lesions or surgery of the axillary in the past few years; 5 . Previous limited movement of the shoulder joint; 6. Agreement to sign the informed consent form.

\section{Intervention}

The specific methods of massage manipulation were as follows: First, the patient was placed in the supine position and a trained physiotherapist performed relaxation training with kneading of the affected limb for 2 minutes. Second, the physiotherapist stripped the cording tissue or subcutaneous nodules with their thumb until the cording and nodules disappeared. Third, the shoulder joint was moved passively over a small range for five times. Finally the shoulder was manipulated passively to achieve flexion, abduction, and extension in order to stretch the affected upper limb. Each session of stretching was maintained for $60 \mathrm{~s}$, which proved beneficial for loosening the adhesion tissue around the shoulder joint and increasing the range of motion. The above intervention as hospitalized therapy was carried out for $20 \mathrm{~min}$ each day for a total of 7 days.

RGS was provided to the resident by telephone contact or at the outpatient department once every week for a total of nineweeks. The specific content of the guidance was as follows: 1. Follow-up of the functional status of the affected upper 
limbs of the patient. 2. Instruct the patient, with the assistance of other family members, to massage the affected limbs and passively maintain the ROM of the shoulder joints three times per day for 15 mins each session.

\section{Outcome measures}

This study used Range of motion(ROM), Visual Analogue Score(VAS), Disabilities of Arm, Shoulder and Hand Scale(DASH), and Ability Of Daily Life(ADL) as the variables to evaluate upper limb function and symptoms. The primary outcome to evaluate joint movement of the upper limbs was the ROM from baseline to 3, 6, and 9 weeks, that included shoulder anteflexion $\left(0 \sim 170^{\circ}\right)$, abduction $\left(0 \sim 170^{\circ}\right)$, extension $\left(0 \sim 60^{\circ}\right)$, external rotation $\left(0 \sim 90^{\circ}\right)$ and internal rotation $\left(0 \sim 70^{\circ}\right)$.

The secondary outcomes were VAS to evaluate the pain of the affected limbs; DASH to evaluate the function of the affected upper limb, with a total score of 100 and lower scores indicating better function[21]; and ADL to assess daily life self-care ability at baseline and 3,6 , and 9 weeks after the start of intervention.

\section{Statistical methods}

Statistical analysis was performed using statistical software SPSS 26.00. The measurement data obtained in this study are expressed as mean(SD), and the comparison of the enumeration data in the general data of patients between groups adopts the chi-square test. The measurement data (ROM, ADL, VAS, DASH) between the treatment group and the control group were compared by independent sample t test. $\mathrm{P}<0.05$ indicates a significant difference and statistically significant. The post hoc analysis was conducted to determine the power of this study using G*Power, version 3.1.9 (Heinrich-Heine-Universitat Dusseldorf, Dusseldorf, Germany).

\section{Results}

A total of 106 participants with post-mastectomy AWS were enrolled in the study(which would in a prospective equivalence trial setting correspond to around $60 \%$ power). Of these patients, 20 were excluded, 12 because they did not meet the inclusion criteria, while 8 refused to participate in the follow-up assessments. In group STMM + RGS group, 2 patients were lost to follow-up at 3 weeks, 2 at 6 weeks, and 1 at 9 weeks. In T-STMM + RGS group, 1 patient was lost to follow-up at 3 weeks and 1 at 9 weeks (Fig. 1). Finally, 41 patients in STMM + RGS group and 38 patients in T-STMM + RGS group completed the 9-weeks of follow-up.

\section{Clinical characteristics}

The clinical characteristics of the patients in the study are shown in Table 1. The major baseline characteristics of the two groups were comparable. The mean (SD) age of all the participants was 50.6 (9.52) years with a range of 28-69 years. Height ranged from 149-168 cm, weight from $42.5-70.0 \mathrm{~kg}$, and BMI from 17.1-28.8 kg/m². The cancer was present in the right breast in 21 patients in STMM + RGS group and 20 in the T-STMM + RGS group, and in the left breast in 20 patients inSTMM + RGS group, and 18 in T-STMM + RGS group.

All the patients in the study had received a modified radical mastectomy, with 10 in STMM + RGS group and 5 in T-STMM + RGS group had a SLND. Of the 31 patients in the STMM + RGS group had an ALND, 9 received low lymph node dissection, 4 medium lymph node dissection, 11 low and medium lymph node dissection, and 7 medium and high lymph node dissection, while 28 patients in T-STMM + RGS group had an ALND, 5 received low lymph node dissection; 3 received medium lymph node dissection; 14 received low and medium lymph node dissection; 2 received high lymph node dissection; 4 received medium and high lymph node dissection.

The number of patients with positive ER and PR receptors was 26 and 17 in STMM + RGS group and 13 and 10 in T-STMM + RGS group, respectively. The patients received different chemotherapy regimens, with the Epirubicin + cyclophosphamide (EC), 
Taxotere + Adriamycin + Andorsun(TAC), Adriamycin + cyclophosphamide + paclitaxe(ECT), Paclitaxel + capecitabine(TX),

Docetaxel + Cyclophosphamide(TC) methods used in 3, 8, 27, 0, and 0 patients in group1, and 0, 10, 21, 1, and 1 patients in TSTMM + RGS group, respectively. A history of diabetes, hypertension, or hyperlipidemia was 6, 7, and 10 in STMM + RGS group, and 3, 1, and 6 in T-STMM + RGS group, respectively.

The anteflexion, abduction, extension, external rotation and internal rotation range of motion mean(SD) are 94.45(26.84), 87.71(24.82, 36.34(12.26), 88.63(6.30), 68.73(6.45), with no statistical difference. The baseline mean(SD) of VAS, ADL and DASH are 2.81(1.46), 91.22(5.17), 34.02(11.42) respectively, with no statistical difference.

Table 1 Baseline demographic and clinical characteristics 


\begin{tabular}{|c|c|c|c|c|c|c|c|c|}
\hline \multicolumn{2}{|l|}{ Characteristic } & $\begin{array}{l}\text { STMM+RGS } \\
\text { group }(n=41) \\
\text { Mean(SD) }\end{array}$ & \multicolumn{2}{|c|}{$\begin{array}{l}\text { T-STMM+RGS } \\
\text { group }(n=38) \\
\text { Mean }(S D)\end{array}$} & $\begin{array}{l}\text { Total(n=79) } \\
\text { Mean(SD) }\end{array}$ & $\begin{array}{l}p \\
\text { value }\end{array}$ & Min. & Max. \\
\hline \multicolumn{2}{|l|}{ Age(year) } & 49.7(8.47) & \multicolumn{2}{|c|}{$51.5 \pm 10.58$} & $50.6(9.52)$ & $p=0.40$ & 28 & 69 \\
\hline \multicolumn{2}{|l|}{ Height(cm) } & $158.78(4.60)$ & \multicolumn{2}{|c|}{$157.87(4.00)$} & $158.34(4.32)$ & $p=0.35$ & 149 & 168 \\
\hline \multicolumn{2}{|l|}{ Weight(kg) } & $56.35(7.49)$ & \multicolumn{2}{|c|}{$57.55(6.17)$} & $56.93(6.87)$ & $p=0.44$ & 42.5 & 70 \\
\hline \multicolumn{2}{|l|}{$\mathrm{BMI}\left(\mathrm{kg} / \mathrm{m}^{2}\right)$} & $22.3(3.26)$ & \multicolumn{2}{|c|}{$22.6(2.33)$} & $22.6(2.83)$ & $p=0.85$ & 17.1 & 28.8 \\
\hline & & \multicolumn{2}{|c|}{ STMM+RGS group $(n=41)$} & \multicolumn{3}{|c|}{ T-STMM+RGS group $(n=38)$} & & \\
\hline \multicolumn{2}{|c|}{ Side of breast cancer(Left; Right) } & \multicolumn{2}{|l|}{$(20 ; 21)$} & \multicolumn{3}{|l|}{$(18 ; 20)$} & & \\
\hline \multicolumn{9}{|l|}{ Surgery type $\mathbb{Z}$} \\
\hline \multicolumn{2}{|l|}{ MRM+SLND } & \multicolumn{2}{|l|}{10} & \multicolumn{3}{|l|}{5} & & \\
\hline \multirow{5}{*}{ ALND } & MRM+LLND & \multicolumn{2}{|l|}{9} & \multicolumn{3}{|l|}{5} & & \\
\hline & MRM+MLND & \multicolumn{2}{|l|}{4} & \multicolumn{3}{|l|}{3} & & \\
\hline & MRM+L/MLND & \multicolumn{2}{|l|}{11} & \multicolumn{3}{|l|}{14} & & \\
\hline & MRM+HLND & \multicolumn{2}{|l|}{0} & \multicolumn{3}{|l|}{2} & & \\
\hline & $\mathrm{MRM}+\mathrm{M} / \mathrm{HLND}$ & 7 & & 4 & & & & \\
\hline Unclear & & 0 & & 5 & & & & \\
\hline ER Receptor(+) & & 26 & & 13 & & & & \\
\hline PR Receptor(+) & & 17 & & 10 & & & & \\
\hline & EC & 3 & & 0 & & & & \\
\hline & TAC & 8 & & 10 & & & & \\
\hline Chemotherapy & ECT & 27 & & 21 & & & & \\
\hline & TX & 0 & & 1 & & & & \\
\hline & $\mathrm{TC}$ & 0 & & 1 & & & & \\
\hline & Unclear & 3 & & 5 & & & & \\
\hline History of diabe & & 6 & & 3 & & & & \\
\hline History of hyper & ension & 7 & & 1 & & & & \\
\hline History of hyper & ipidemia & 10 & & 6 & & & & \\
\hline Primary outcom & & $\begin{array}{l}\text { STMM+RGS } \\
\operatorname{aroup}(n=41)\end{array}$ & $\begin{array}{l}\text { T- } \\
\text { STMM+R }\end{array}$ & Saroun & Total $(\mathrm{n}=79)$ & & $p$ value & \\
\hline & & Mean(SD) & $(\mathrm{n}=38) \mathrm{Me}$ & & Mean(SD) & & & \\
\hline & Anteflexion $\left(0 \sim 170^{\circ}\right)$ & $94.00(26.62)$ & $94.94(27$ & 43) & $94.45(26.84)$ & & $p=0.89$ & \\
\hline & Abduction $\left(0 \sim 170^{\circ}\right)$ & 88.85(23.99) & $86.47(25$ & 95) & $87.71(24.82)$ & & $p=0.67$ & \\
\hline RUIVI & Extension $\left(0 \sim 60^{\circ}\right)$ & $34.96(13.52)$ & $37.83(10$ & 71) & $36.34(12.26)$ & & $p=0.30$ & \\
\hline & $\begin{array}{l}\text { External } \\
\text { rotation }\left(0 \sim 90^{\circ}\right)\end{array}$ & 88.18(8.35) & $89.12(2.7$ & & $88.63(6.30)$ & & $p=0.51$ & \\
\hline
\end{tabular}




\begin{tabular}{|lllll|}
\multicolumn{1}{|c}{$\begin{array}{l}\text { Internal } \\
\text { rotation(0 70 })\end{array}$} & $68.10(8.55)$ & $69.42(2.14)$ & $68.73(6.45)$ & $p=0.36$ \\
\hline VAS(pain score 1-10) & $2.83(1.34)$ & $2.78(1.60)$ & $2.81(1.46)$ & $p=0.90$ \\
\hline ADL(score 0-100) & $91.46(5.15)$ & $90.96(5.25)$ & $91.22(5.17)$ & $p=0.73$ \\
\hline DASH(score 0-58.3) & $31.97(10.16)$ & $36.23(12.40)$ & $34.02(11.42)$ & $p=0.10$ \\
\hline
\end{tabular}

MRM- Modified radical mastectomy; SLND- Sentinel lymph node dissection; LLND- Low lymph node dissection; MLNDMedian lymph node dissection; L/MLND- Low and Median lymph node dissection; HLND- High lymph node dissection; M/HLND- Median and high lymph node dissection; ROM- Range of motion; ADL- Barthel score; DASH- Disabilities of Arm, Shoulder and Hand Scale. EC- Epirubicin+cyclophosphamide; TAC- Taxotere+Adriamycin+Andorsun; ECT-

Adriamycin+cyclophosphamide+paclitaxel; TX-Paclitaxel+capecitabine; TC- Docetaxel+Cyclophosphamide.

\section{Primary Outcomes}

As shown in Table 2, after 3 weeks of treatment, anteflexion and abduction ROM have a statistical difference between the STMM + RGS group and T-STMM + RGS group group with mean(SD): $116.06(27.64), 95 \% \mathrm{Cl} 1.74$ to 25.89, $p=0.03$;

$106.56(29.96), 95 \% \mathrm{Cl} 0.38$ to $24.07, \mathrm{p}=0.04$, respectively. A significant difference in ROM was also observed between the two groups after 6 weeks with meann(SD):128.49(23.87), $95 \% \mathrm{Cl} 10.63$ to $35.18, p=0.00 ; 120.00(29.18), 95 \% \mathrm{Cl} 5.89$ to $30.87 p=$

$0.01 ; 49.69(19.06), 95 \% \mathrm{Cl} 0.84$ to $9.36, p=0.02$, and 9 weeks of treatment with mean(SD): $141.10(27.24), 95 \% \mathrm{Cl} 8.65$ to 31.49 , $p=0.001 ; 135.51(28.27), 95 \% \mathrm{Cl} 7.83$ to $31.74, p=0.001 ; 54.74(7.32), 95 \% \mathrm{Cl} 1.08$ to $7.40, p=0.009$.

\section{Secondary outcome}

After 6 and 9 weeks of treatment, VAS was significantly different between the two groups with mean(SD): $1.10(1.30), 95 \% \mathrm{Cl}$ -1.28 to $-0.16 p=0.01 ; 0.65(0.97), 95 \% \mathrm{Cl}-0.90$ to $-0.04, p=0.03$, respectively. As shown in Table 2 , after 3 and 6 weeks of treatment, ADL was significantly different between STMM + RGS group and T-STMM + RGS group with mean(SD): 96.27(4.49), $95 \% \mathrm{Cl} 0.22$ to $4.15, p=0.03 ; 98.22(3.21), 95 \% \mathrm{Cl} 0.80$ to $3.52 p=0.02$, respectively.. DASH was also significantly different in the two groups after 3,6 , and 9 weeks of treatment with mean(SD): $23.93(12.12), 95 \% \mathrm{Cl}-15.55$ to $-5.73, p=0.00 ; 18.68(9.68)$, $95 \% \mathrm{Cl}-18.04$ to $-8.58, p=0.00 ; 13.36(10.69), 95 \% \mathrm{Cl}-15.84$ to $-7.81, p=0.00$ respectively.

Table 2. Comparison of changes in pain, ROM, barthel, DASH score at $3 \square 6 \square 9$ weeks follow up. 


\begin{tabular}{|c|c|c|c|c|c|c|}
\hline \multirow{2}{*}{\multicolumn{2}{|c|}{ Outcomes }} & \multirow{2}{*}{$\begin{array}{l}\text { STMM+RGS } \\
\text { group }(n=41) \\
\text { Mean(SD) }\end{array}$} & \multirow{2}{*}{$\begin{array}{l}\text { T- } \\
\text { STMM+RGS group }(n=38) \text { Mean(SD) }\end{array}$} & \multicolumn{2}{|c|}{ Between-group difference } & \multirow[t]{2}{*}{$P$ value } \\
\hline & & & & Mean(SD) & $95 \% \mathrm{Cl}$ & \\
\hline \multicolumn{7}{|c|}{$\begin{array}{l}\text { At } 3 \text { weeks follow } \\
\text { up }\end{array}$} \\
\hline \multirow{5}{*}{ ROM } & Anteflexion & $122.71(27.54)$ & $108.89(26.25)$ & $116.06(27.64)$ & $1.74 ; 25.89$ & $p=0.03^{*}$ \\
\hline & Abduction & $112.44(25.95)$ & $100.21(26.92)$ & $106.56(29.96)$ & $0.38 ; 24.07$ & $p=0.04^{\star}$ \\
\hline & Extension & 43.43(11.96) & $43.03(9.63)$ & 43.24(10.83) & $-4.49 ; 5.29$ & $p=0.87$ \\
\hline & $\begin{array}{l}\text { External } \\
\text { rotation }\end{array}$ & 86.32(13.79) & $87.97(5.96)$ & 87.11(10.73) & $-6.42 ; 3.17$ & $p=0.50$ \\
\hline & $\begin{array}{l}\text { Internal } \\
\text { rotation }\end{array}$ & $67.73(9.00)$ & 69.02(3.08) & $68.35(6.82)$ & $-4.35 ; 1.74$ & $p=0.21$ \\
\hline \multicolumn{2}{|c|}{ VAS(pain score) } & $1.46(1.16)$ & $1.78(1.60)$ & $1.61(1.39)$ & $-0.95 ; 2.30$ & $p=0.30$ \\
\hline \multicolumn{2}{|l|}{$A D L$} & 97.32(3.89) & $95.13(4.86)$ & $96.27(4.49)$ & $0.22 ; 4.15$ & $p=0.03^{*}$ \\
\hline \multicolumn{2}{|c|}{ DASH } & 18.81(9.91) & 29.45(11.97) & 23.93(12.12) & $\begin{array}{l}-15.55 \\
-5.73\end{array}$ & $p=0.00 \#$ \\
\hline \multicolumn{7}{|c|}{$\begin{array}{l}\text { At } 6 \text { weeks follow } \\
\text { up }\end{array}$} \\
\hline \multirow{5}{*}{ ROM } & Anteflexion & 139.51(29.19) & $116.61(25.24)$ & $128.49(23.87)$ & $10.63 ; 35.18$ & $p=0.00 \#$ \\
\hline & Abduction & $128.85(30.12)$ & $110.47(25.18)$ & $120.00(29.18)$ & $5.89 ; 30.87$ & $p=0.01^{*}$ \\
\hline & Extension & $52.14(10.46)$ & 47.04(8.34) & 49.69(19.06) & $0.84 ; 9.36$ & $p=0.02^{*}$ \\
\hline & $\begin{array}{l}\text { External } \\
\text { rotation }\end{array}$ & 86.21(13.31) & $87.89(5.80)$ & 87.02(11.16) & $-6.34 ; 2.99$ & $p=0.48$ \\
\hline & $\begin{array}{l}\text { Internal } \\
\text { rotation }\end{array}$ & $66.22(13.20)$ & $68.23(4.70)$ & 67.19(10.31) & $-6.55 ; 2.46$ & $p=0.37$ \\
\hline \multicolumn{2}{|c|}{ VAS(pain score) } & $0.76(1.02)$ & $1.47(1.47)$ & $1.10(1.30)$ & $-1.28 ;-0.16$ & $p=0.01^{*}$ \\
\hline \multicolumn{2}{|l|}{ ADL } & $99.26(2.11)$ & $97.10(3.80)$ & $98.22(3.21)$ & $0.80 ; 3.52$ & $p=0.02^{\star}$ \\
\hline \multicolumn{2}{|c|}{ DASH } & $12.28(9.06)$ & $25.59(11.47)$ & 18.68(9.68) & $\begin{array}{l}-18.04 \\
-8.58\end{array}$ & $p=0.00 \#$ \\
\hline \multicolumn{7}{|c|}{$\begin{array}{l}\text { At } 9 \text { weeks follow } \\
\text { up }\end{array}$} \\
\hline \multirow{5}{*}{ ROM } & Anteflexion & $150.76(25.57)$ & $130.68(25.35)$ & $141.10(27.24)$ & $8.65 ; 31.49$ & $p=0.001 \#$ \\
\hline & Abduction & $145.02(27.76)$ & $125.24(25.43)$ & $135.51(28.27)$ & $7.83 ; 31.74$ & $p=0.001 \#$ \\
\hline & Extension & 56.78(7.05) & $52.53(7.05)$ & $54.74(7.32)$ & $1.08 ; 7.40$ & $p=0.009 \#$ \\
\hline & $\begin{array}{l}\text { External } \\
\text { rotation }\end{array}$ & 85.85(13.07) & $87.42(6.05)$ & $86.60(10.28)$ & $-1.57 ; 2.32$ & $p=0.50$ \\
\hline & $\begin{array}{l}\text { Internal } \\
\text { rotation }\end{array}$ & $67.27(7.98)$ & $68.03(4.30)$ & $67.64(6.45)$ & $-3.66 ; 2.14$ & $p=0.61$ \\
\hline \multicolumn{2}{|c|}{ VAS(pain score) } & $0.43(0.84)$ & $0.89(1.06)$ & $0.65(0.97)$ & $-0.90 ;-0.04$ & $p=0.03^{*}$ \\
\hline \multicolumn{2}{|l|}{$A D L$} & $99.63(1.73)$ & $99.21(1.85)$ & $99.43(1.79)$ & $-0.38 ; 1.22$ & $p=0.30$ \\
\hline \multicolumn{2}{|c|}{ DASH } & 7.67(7.12) & 19.50(10.57) & 13.36(10.69) & $\begin{array}{l}-15.84 \\
-7.81\end{array}$ & $P=0.00 \#$ \\
\hline
\end{tabular}


ROM- Range of motion; ADL- Barthel score; DASH- Disabilities of Arm, Shoulder and Hand Scale. SD- standard deviation; 95\% Cl- 95\% confifidence intervals; $\boldsymbol{p}$ Value from the superiority trial using a two-sided test hypothesis; ${ }^{*}-p<0.05 ; \#-p<0.01$.

\section{Discussion}

This study shows that STMM combined with RGS can improve anteflexion, abduction, and extension of shoulder ROM, relieve upper limb pain, improve the function of the affected upper limb and the ability of patients' to self-care in the early stage of AWS. However, long-term ability to self-care showed no significant improvement. The study also showed that edema around the AWS cord was alleviated by musculoskeletal ultrasound(Picture 2).

There are only a small number of reports on AWS treatment, although a research focus on this treatment has increased gradually in recent years. Cho[20] used physical therapy combined with manual lymphatic drainage to treat AWS patients and showed that DASH, pain, and the quality-of-life score of patients improved after four weeks of treatment. Fourie[22] also reported that stripping the cord tissue of AWS patients for three weeks increased the flexion and abduction of the shoulder joint. The results of these two studies therefore suggest that strip massage and limb exercise may alleviate the symptoms of AWS.

The intervention pattern used in our study was different from that used in earlier studies. In our country, the postoperative rehabilitation of breast cancer is not well understood and there is low acceptance of the procedure, resulting in poor patient compliance. The current study treated patients with STMM + RGS using a clinical intervention period of one week, which is shorter than that used in previous research interventions. Unexpectedly, STMM + RGS achieved clinical effects, a finding that demonstrates a patient's function can be improved according to the method of hospitalization therapy and remote rehabilitation. Early manual intervention ameliorated postoperative joint movement, upper limb function, and alleviated pain. Our study included remote follow-up of patients by telephone to provide guidelines for rehabilitation, with this strategy reducing the length of hospital stay and associated costs for patients.

The second difference of our study was the intervention method used- the massage manipulation- which improved on the basis of previous research to treat the AWS in the affected limbs by using massage to strip the cordings and subcutaneous nodules, and supplementary shoulder joint stretching to increase the ROM. This comprehensive treatment resulted in a greater improvement in function than that reported by previous studies[20, 22].

After nine weeks of follow-up after intervention we showed that the ROM improved significantly compared to that achieved in the T-STMM + RGS group. However, a small number of patients had limited internal and external rotation ROM during the nine weeks of follow-up and this may explain the insignificant difference in ROM we observed between the two groups. In addition, the pain scores of patients in STMM + RGS group were not significantly different from those in T-STMM + RGS group during 3weeks of follow-up, although during 6- and 9-weeks of follow-up the scores were significantly lower in the STMM + RGS group than in the T-STMM + RGS group. This is a different result than that reported by Cho[20]. A possible reason for this difference may be that the axillary cording and nodules of AWS patients were not diminished in the early stages of treatment and therefore the pain persisted despite an apparent treatment. Our study also carried out comprehensive assessment of the patients' affected upper limb function and ability to self-care, and found that the DASH disability score improved significantly after 3,6 , and 9 weeks of follow-up compared with that recorded in the T-STMM + RGS group. In contrast, the ADL showed no significant improvement at the 9th week of follow-up. ADL evaluation of upper limb function is limited which may account for the minimal improvement in ADL score with no statistical difference between the two groups being observed. Taken together, these results suggest that the use of STMM + RGS can significantly improve a patient's upper limb function and reduce the risk of disability, but does not significantly improve the patient's long-term ability to daily self-care.

During the clinical intervention we found that patients experienced obvious pain during the treatment to strip the cordings and nodules, with some patients suffering unbearable pain. However, after the cordings and nodules were stripped completely the pain on the affected side was gradually relieved and joint ROM increased. However, it remains unclear whether this massage technique promotes inflammation of the affected limb as according to Koehler[23] the 'cording' is considered to be a blood 
vessel and/or a lymphatic channel. Notwithstanding this possibility, musculoskeletal ultrasound examination in our study showed that edema obviously reduced after treatment with no adverse events observed after nine weeks of follow-up. We also observed that there are two manifestations of the AWS during clinical intervention. The first is that the texture of the cording is more brittle and easy to strip. During implementation of the method we were able to hear the obvious sound of the cording breaking. The second manifestation is that the cording may be difficult to strip and in these patients no significant improvement in joint mobility was observed after the end of the massage treatment. However, whether there is a difference in the long-term prognosis of these two types of AWS patients remains unclear because our study did not include a rigorous data analysis of this possibility. Our study also used musculoskeletal ultrasound that showed the echo of the cording was similar to that of fat tissue, although the nature of this recording was unclear and therefore requires further investigation.

This study had several limitations. First, with a sample size of 79 the difference in the TT-RA between the two groups could only be found at a power of $60 \%$ and therefore a larger sample size is needed to test the effectiveness of the intervention. Second, the study only followed-up the patients for 9 weeks after the intervention and did not analyze long-term efficacy. A study with a long period of follow-up is therefore necessary. Finally, the study only evaluated the patient's shoulder joint ROM, pain, upper limb function, and daily living ability, and did not analyze other symptoms associated with AWS such as numbness and tightness. Although the patient's upper limb numbness and tightness were followed-up during the course of treatment, changes in these two indicators were difficult to quantify and accordingly the results of the analyses were not included in this article.

In conclusion, the understanding and management of AWS by both clinical workers and patients' needs to be improved, with effective identification of the similarities and differences between AWS and other complications after breast cancer surgery. Early intervention and treatment of AWS needs to be achieved to obtain good clinical outcomes. Although the results of the current study need to be verified further by clinical studies with a larger sample size, the preliminary results indicate that early use of STMM and RGS in AWS patients improves the flexion, abduction, and extension ROM of shoulder joints, in addition to relieving pain of the upper limb on the affected side, and improving upper limb function. Based on these findings we propose a new treatment strategy for clinical management of AWS. Research on the mechanism and evaluation of AWS is limited and therefore further research is needed to fully understand the mechanism and to set-up better methods for evaluating AWS so that clinicians are able to diagnose and treat the symptoms to a high clinical standard.

\section{Declarations}

Funding: The reported work was supported in part by research grants from the Chongqing Health Commission and Science and Technology Bureau joint research project of Traditional Chinese medicine (2019ZY3408).

Conflicts of Interest: none.

Acknowledgements: The authors would like to express their gratitude to EditSprings (https://www.editsprings.com/) for the expert linguistic services provided.

Ethics approval and consent to participate: The study has been approved by the Ethics Committee of the First Affiliated Hospital of Chongqing Medical University and registered in the clinical trial registration center, the registration number is ChiCTR1900028060. The patients enrolled received oral and written information about the study and written informed consent forms.

Availability of data and materials: All the patients were from the Department of Endocrinology and Breast Surgery, the First Affiliated Hospital of Chongqing Medical University.

Consent for publication: The authors consent for publication of the study.

Competing interests: The authors declare that they have no competing interests. 
Funding:The reported work was supported in part by research grants from the Chongqing Health Commission and Science and Technology Bureau joint research project of Traditional Chinese medicine (2019ZY3408).

Authors' contribution: Jia Qia responsible for article data collection and writing the paper, Qi-hui Yang, Jing Zhan and Yi Li assist the data collection. Yu-han Kong and Ding-qun Bai responsible for the revise of the paper.

Acknowledgements: The authors would like to express their gratitude to EditSprings (https://www.editsprings.com/) for the expert linguistic services provided.

\section{References}

1. Li T, Mellp-Thoms C, Brennan PC (2016) Descriptive epidemiology of breast cancer in China: incidence, mortality, survival and prevalence[J]. Breast cancer research treatment 159(3):395-406

2. Schuitevoerderd, White I, Fortino J et al (2016) Axillary web syndrome: an underappreciated complication of sentinel node biopsy in melanoma[J]. Am J Surg 211(5):846-849

3. Lee KC, W CY, Chen CP et al (2018) Axillary web syndrome following epidermal inclusion cyst: a case report and literature review[J]. Clin Exp Dermatol 44(1):64-66

4. Horick N, O'Toole J et al (2012) Sentinel lymph node biopsy at the time of mastectomy does not increase the risk of lymphedema: implications for prophylactic surgery[J]. Breast Cancer Res Treat 135(3):781-789

5. Yeung WM, McPhail SM, Kuys SS (2015) A systematic review of axillary web syndrome (AWS)[J]. J Cancer Surviv 9(4):576-598

6. Leidenius M, Leppanen E, Krogerus L, von Smitten K (2003) Motion restriction and axillary web syndrome after sentinel node biopsy and axillary clearance in breast cancer[J]. Am J Surg 185(2):127-130

7. Figueira PVG, Haddad CAS, De Almeida Rizzil SKL et al (2018) Diagnosis of Axillary Web Syndrome in Patients After Breast Cancer Surgery: Epidemiology, Risk Factors, and Clinical Aspects: A Prospective Study[J]. Am J Clin Oncol 41(10):992-996

8. O'Toole J, Miller CL, Specht MC, Skolny MN, Jammallo LS, Horick N et al (2013) Cording following treatment for breast cancer[J]. Breast Cancer Res Treat 140(1):105-111

9. Bergmann A, Mendes VV, de Almeida Dias R, do Amaral e Silva B, da Costa Leite Ferreira MG, Fabro EAN. Incidence and risk factors for axillary web syndrome after breast cancer surgery[J]. Breast Cancer Res Treat, 2012, 131(3):987-992

10. Rashtak S, Gamble GL, Gibson LE, Pittelkow MR (2012) From furuncle to axillary web syndrome: shedding light on histopathology and pathogenesis[J]. Dermatology 224:110-114

11. Huang Hlhylyctcyc (2017) The upper-limb volumetric changes in breast cancer survivors with axillary web syndrome[J]. Eur J Cancer Care 26(2):26-37

12. Aydogan F, Belli AK, Bağhaki S, Karabulut K, Tahan G, Uras C (2008) Axillary web syndrome after sentinel node biopsy[J]. Breast Care (Basel) 3(4):277-278

13. Koehler LA, Hunter DW, Blaes AH et al (2018) Function, Shoulder Motion, Pain, and Lymphedema in Breast Cancer With and Without Axillary Web Syndrome: An 18-Month Follow-Up[J]. Phys Ther 98(96):518-527

14. McLaughlin SA, Bagaria S, Gibson T, Arnold M, Diehl N, Crook J et al (2013) Trends in risk reduction practices for the prevention of lymphedema in the first 12 months after breast cancer surgery[J]. J Am Coll Surg 216(3):380-389

15. Galantino ML, Stout NL (2013) Exercise interventions for upper limb dysfunction due to breast cancer treatment[J]. Phys Ther 93(10):1291-1297

16. McNeely ML, Binkley JM, Pusic AL, Campbell KL, Gabram S, Soballe PW (2012) A prospective model of care for breast cancer rehabilitation: postoperative and postreconstructive issues[J]. Cancer 118(S8):2226-2236

17. Miller CL, Specht MC, Skolny MN, Jammallo LS, Horick N, O’Toole J et al (2012) Sentinel lymph node biopsy at the time of mastectomy does not increase the risk of lymphedema: implications for prophylactic surgery[J]. Breast Cancer Res Treat

Page $11 / 14$ 
135(3):781-789

18. Lattanzi JB, Zimmerman A, Marshall LM (2012) Case report of axillary web syndrome[J]. Rehabil Oncol 30(1):18-21

19. Tilley A, Thomas-MacLean R, Kwan W (2009) Lymphatic cording or axillary web syndrome after breast cancer surgery[J]. Can J Surg 52(4):105-106

20. Youngki Cho J, Do S, Jung et al (2016) Effects of a physical therapy program combined with manual lymphatic drainage on shoulder function, quality of life, lymphedema incidence, and pain in breast cancer patients with axillary web syndrome following axillary dissection[J]. Support care cancer 24(5):2047-2057

21. Beaton DE, Katz JN, Fossel AH et al (2001) Measuring the whole or the parts validity, reliability, and responsiveness of the disabilities of the arm, shoulder and hand outcome measure in different regions of the upper extremity[J]. Hand Ther 142(2):128-146

22. Fourie WJ, Robb KA (2009) Physiotherapy management of axillary web syndrome following breast cancer treatment: Discussing the use of soft tissue techniques[J]. Physiotherapy 95(4):314-320

23. Koehler LA. Axillary web syndrome ongoing medical evaluation dissertation[J]. Minneapolis (MI): University of Minnesota; 2013

\section{Figures}



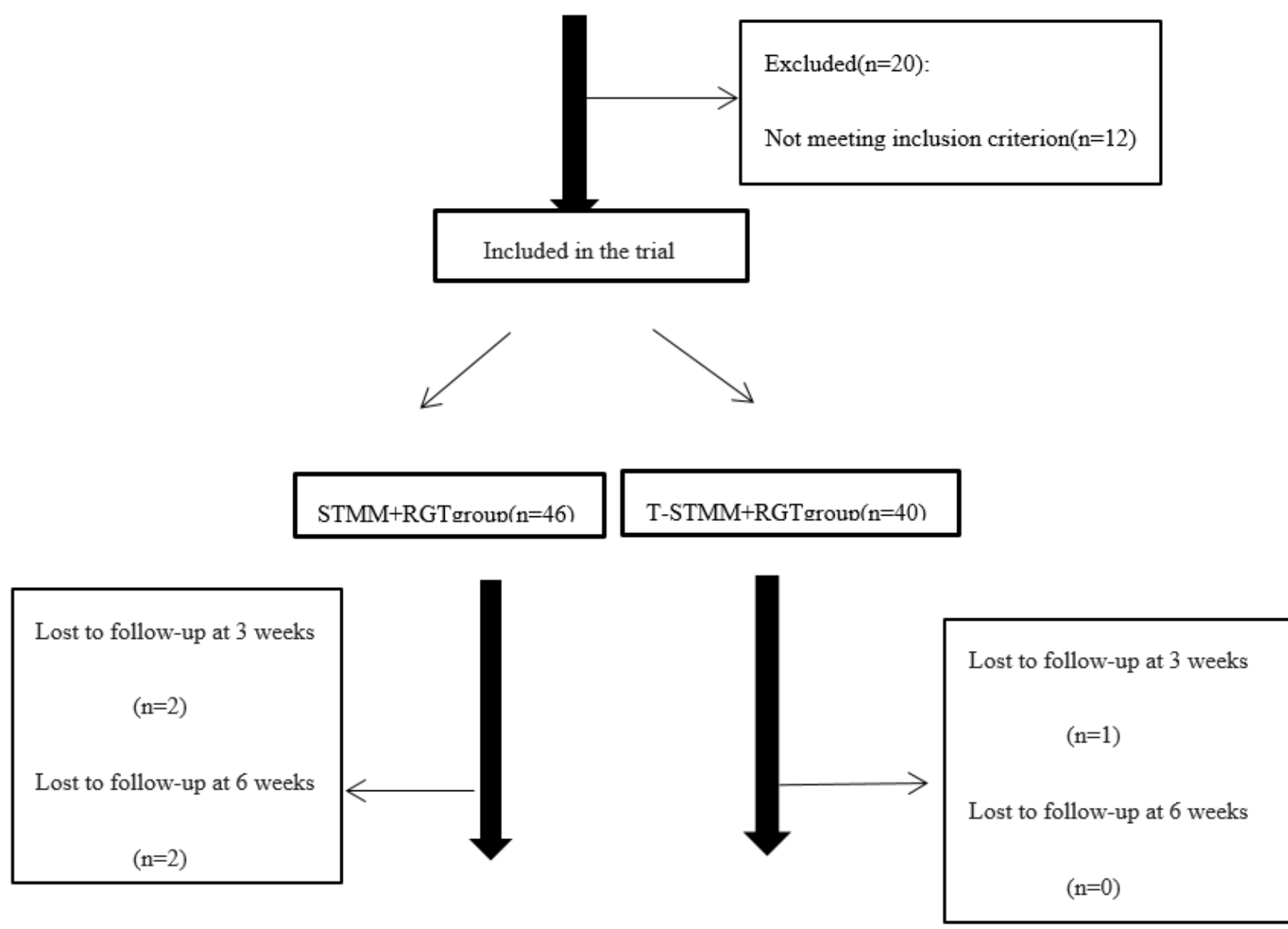

Included in primary
analysis(n=41)

\section{Figure 1}

Flow Chart 

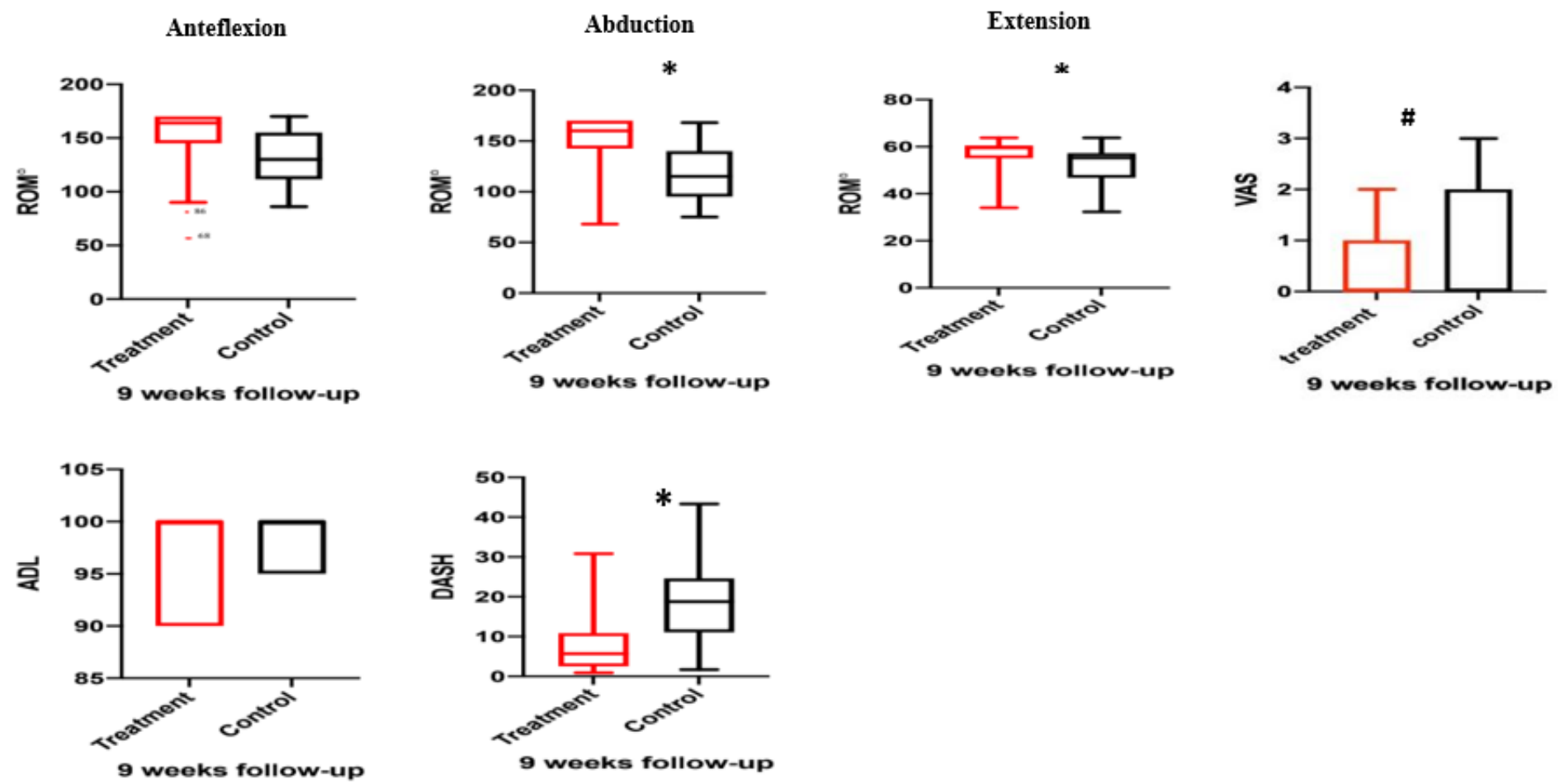

Figure 2

Box plot of outcome indicators at 9 weeks follow-up. p- Value from the superiority trial using a two-sided test hypothesis; *$p<0.05$; \#- $p<0.01$; Treatment- short-term massage manipulation combine with remote guided self-exercise group(STMM+RGS group), Control- the control group(T-STMM+RGS group).

\section{Supplementary Files}

This is a list of supplementary files associated with this preprint. Click to download.

- Picture1.png

- Picture2.png 